\title{
OPTIMAL AEROASSISTED TRANSFER BETWEEN COPLANAR ELLIPTICAL ORBITS $\dagger$
}

\author{
Nguyen X. Vinh and Jennie R. Johannesen \\ Department of Aerospace Engineering, The University of Michigan, Ann Arbor, MI 48109-2140, U.S.A.
}

(Received 28 January 1986)

\begin{abstract}
This paper presents the solution for minimum-fuel, free-time transfer between coplanar elliptical orbits with the possible use of a planetary atmosphere to generate a decelerative braking force. The optimal pure propulsive two-impulse transfer is first considered. It is shown that the solution is obtained by solving a set of three equations for three unknowns. Reduction of the general equations is made for the case of symmetrical transfer and a complete first-order solution is provided for the case of transfer from nearly circular orbit. In aeroassisted transfer atmospheric braking at the perigee can be used to circularize the orbit, and in the circular configuration the orbit can be arbitrarily rotated without fuel consumption. It is shown that complete circularization of this intermediary orbit is optimal only when the rotation angle is large, and an explicit formula for evaluating this critical angle is provided. A complete solution is presented for the case of optimal rotation of an orbit. Finally, an example of optimal aeroassisted transfer is provided for the case of a transfer from a low-energy orbit to a high-energy orbit.
\end{abstract}

\section{INTRODUCTION}

With the advent of the Orbital Transfer Vehicle (OTV), there is a current research interest in the analysis of combined aerodynamic and propulsive maneuver as a technique for reduction of the fuel consumption in orbital transfer. The classical results have been presented in two recent survey papers[1,2]. As is usually the case for pioneering work, up to now the authors concentrated their efforts on the cases where the use of atmospheric passages is clearly advantageous. These are the case of transfer from a high orbit to a low orbit, and the case of transfer between noncoplanar circular orbits with large plane change. Furthermore, there has been little use of the outstanding results of the theory of optimal space trajectories obtained during the last two decades [3-6].

As a new initiative in the integration of the theory of optimal propulsive transfer into the theory of aeroassisted transfer, it is proposed to solve in this paper the problem of orbital transfer between coplanar elliptical orbits. First, the pure propulsive transfer is analyzed with the restriction of two impulses for the transfer. The optimal switching conditions are reviewed, and it is shown that the solution is obtained by solving a set of three nonlinear equations for three unknowns. Next, in order to show that aeroassisted transfer can still be fuel optimal in the planar case when the energy of the final orbit is equal to the energy of the initial orbit, we consider the problem of planar rotation of an orbit. A semianalytical solution is obtained for the pure propulsive

+Paper presented at the 36 th Congress of the International Astronautical Federation, Stockholm, Sweden, 7-12 October 1985. maneuver. By comparing its optimal solution with a simple scheme for aeroassisted transfer it is shown that, for high eccentricity and rotation angle, aeroassisted transfer is a fuel saving maneuver. In the aeroassisted transfer, only the drag is required. It is used to circularize the orbit, and hence in this intermediary orbit configuration, the rotation of the apsidal line can be achieved without fuel consumption. Several graphs are generated showing different regions of optimality. In the next step, it is shown that in the optimal aeroassisted transfer, complete circularization of the intermediate orbit is not necessary. An analytical proof is presented, giving an explicit condition for noncircularization. Finally, a complete numerical solution is presented for a case of optimal aeroassisted transfer from a low-energy orbit to a high-energy orbit.

\section{PURE PROPULSIVE TRANSFER}

The theory of optimal propulsive transfer between orbits is very complete and has been masterfully presented by Marec[6] in his monograph on optimal space trajectories. For planar transfer we consider the configuration of the orbits as shown in Fig. 1.

The initial orbit $O_{1}$ and the final orbit $O_{2}$ are given. These are defined by the orbital elements $e_{1}, p_{1}, e_{2}, p_{2}$ and the angle $\alpha$ between the apsidal lines from $O_{1}$ to $O_{2}$. Let $I_{1}$ and $I_{2}$ be the location of the impulses as defined by the true anomalies $\theta_{1}$ and $\theta_{2}$ on the initial and the final orbit, respectively. If $r_{1}$ and $r_{2}$ are the radial distances, we have

$$
r_{1}=\frac{p_{1}}{1+e_{1} \cos \theta_{1}}, \quad r_{2}=\frac{p_{2}}{1+e_{2} \cos \theta_{2}}
$$




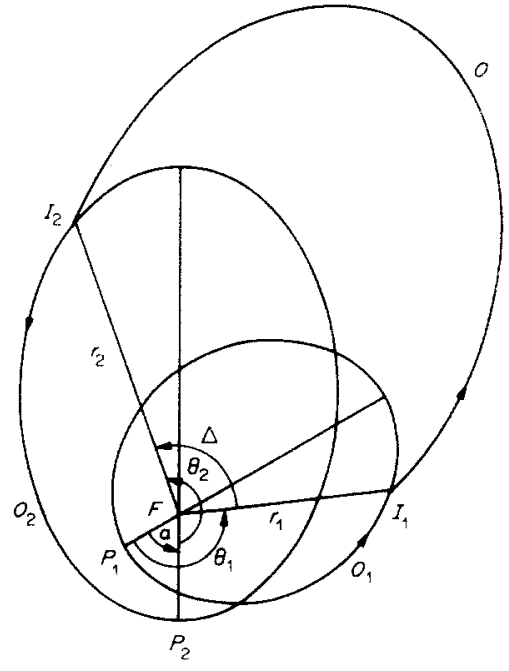

Fig. 1. Configuration of the terminal orbits.

There are three optimal conditions relating the elements at the impulses. They are obtained along the transfer orbit $O$ with unknown elements $e, p$ and $\omega$. Of course, $\omega$ denotes the argument of the perigee of the transfer orbit, measured from the perigee of the initial orbit, Let $\phi_{1}$ and $\phi_{2}$ be the thrust angles measured from the local horizontal (Fig. 2).

Let

$$
S_{i}=\sin \phi_{i}, \quad T_{i}=\cos \phi_{i}
$$

be the direction cosines of the impulses. On the transfer orbit, the impulses are located by the true anomalies $f_{1}$ and $f_{2}$. Let $\Delta$ be the transfer angle. From Figs 1 and 2 , we have

$$
\Delta=f_{2}-f_{1}=\alpha+\theta_{2}-\theta_{1} .
$$

For the present analysis, it is convenient to use the three optimal conditions as obtained by Vinh[4]. They are

$$
\begin{aligned}
\left(2+x_{1}\right) K_{1}-\left(1+x_{1}\right) T_{1}= & \left(2+x_{2}\right) K_{2} \\
& -\left(1+x_{2}\right) T_{2} \\
{\left[y_{1} T_{1}-\left(1+x_{1}\right) S_{1}\right]\left(T_{1}-K_{1}\right)=} & S_{1} T_{1} \\
{\left[y_{2} T_{2}-\left(1+x_{2}\right) S_{2}\right]\left(T_{2}-K_{2}\right)=} & S_{2} T_{2}
\end{aligned}
$$

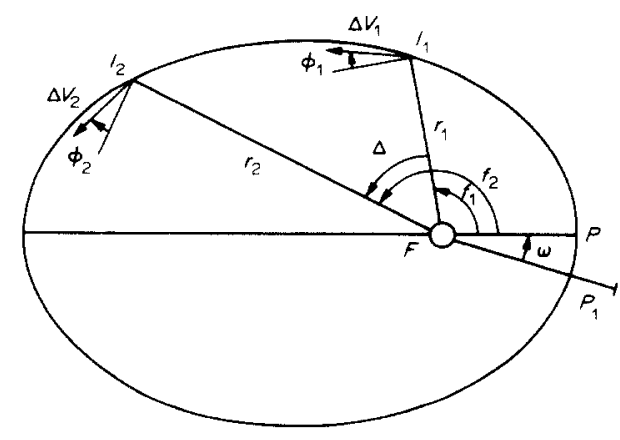

Fig. 2. Impulses on the transfer orbit. where, by definition

$$
\begin{aligned}
1+x_{i} & =1+e \cos f_{i}=\frac{p}{r_{i}}=\frac{p}{p_{i}}\left(1+\epsilon_{i} \cos \theta_{i}\right) \\
y_{1} & =e \sin f_{i} \\
K_{1} & =\frac{S_{2}-S_{1} \cos \Delta}{\sin \Delta}, \quad K_{2}=\frac{S_{2} \cos \Delta-S_{1}}{\sin \Delta}
\end{aligned}
$$

Furthermore, by using the velocity diagram at the impulse, we have the relations

$$
\begin{aligned}
& \tan \phi_{1}=\frac{r_{1}}{\left(\sqrt{p}-\sqrt{p_{1}}\right)}\left(\frac{y_{1}}{\sqrt{p}}-\frac{e_{1}}{\sqrt{p_{1}}} \sin \theta_{1}\right) \\
& \tan \phi_{2}=\frac{r_{2}}{\left(\sqrt{p}-\sqrt{p_{2}}\right)}\left(\frac{y_{2}}{\sqrt{p}}-\frac{e_{2}}{\sqrt{p_{2}}} \sin \theta_{2}\right)
\end{aligned}
$$

It now appears that instead of using eleven unknowns for solving this problem as suggested by Lawden[3], we can simply choose three unknowns namely $\theta_{1}, \theta_{2}$ and $p$, and solve for them using the three eqns (4), (5) and (6). In these equations $\left(1+x_{i}\right)$ are explicit functions of $p$ and $\theta_{i}$ as shown in eqn (7). As for $y_{i}$ they are given explicitly as functions of $p$ and $\theta_{i}$ by the identities

$$
\begin{aligned}
& y_{1}=\tan \frac{\Delta}{2}+\frac{1}{\sin \Delta}\left[\frac{p}{r_{1}} \cos \Delta-\frac{p}{r_{2}}\right] \\
& y_{2}=-\tan \frac{\Delta}{2}+\frac{1}{\sin \Delta}\left[\frac{p}{r_{1}}-\frac{p}{r_{2}} \cos \Delta\right]
\end{aligned}
$$

Upon substituting into eqns (10) and (11), we can evaluate $\tan \phi_{i}$ and hence $S_{i}$ and $T_{i}$. This gives $K_{i}$ as functions of the same three variables. Once the problem has been solved, the eccentricity of the transfer orbit is obtained from

$$
e^{2}=x_{1}^{2}+y_{1}^{2}
$$

while its argument of the perigee is given by

$$
\omega=\theta_{1}-f_{1}, \quad \tan f_{1}=y_{1} / x_{1}
$$

The characteristic velocities required for the transfer are computed from

$$
\begin{aligned}
& \Delta V_{1}=\frac{\sqrt{\mu}}{r_{1} \cos \phi_{1}}\left(\sqrt{p}-\sqrt{p_{1}}\right) \\
& \Delta V_{2}=\frac{\sqrt{\mu}}{r_{2} \cos \phi_{2}}\left(\sqrt{p_{2}}-\sqrt{p}\right)
\end{aligned}
$$

where $\mu$ is the gravitational constant.

Explicit solution can only be obtained for some special cases involving circular orbits or coaxial orbits. Reduction of the equations can also be obtained when the transfer displays a certain elements of symmetry. For the general cases, a numerical program has been written to routinely solve the system. We have also solved the problem in the case where the initial orbit is nearly circular. Its solution to the first order in the small eccentricity is the basis for our proof of noncircularization in aeroassisted transfer. 


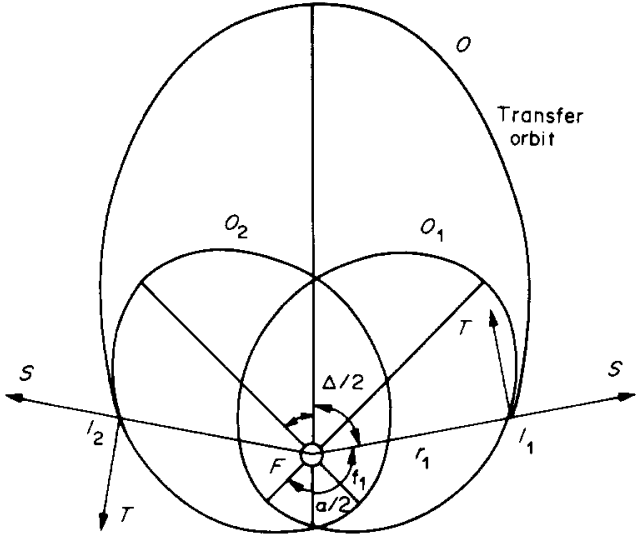

Fig. 3. Symmetric transfer.

\section{OPTIMAL ROTATION OF ORBIT}

We consider the case where the terminal orbits have the same shape and size, that is $e_{2}=e_{1}, p_{2}=p_{1}$. It is proposed to rotate the orbit by the prescribed angle $\alpha$ with minimum fuel consumption.

We first consider the case of pure propulsive transfer.

The transfer is symmetric (Fig. 3) and we trivially have

$$
\begin{aligned}
& \omega=\frac{\alpha}{2}, \quad \theta_{2}=2 \pi-\theta_{1}, \quad S_{2}=S_{1}, \quad T_{2}=-T_{1} \\
& K_{2}=-K_{1}, \quad f_{2}=2 \pi-f_{1}, \quad x_{2}=x_{1}, \quad y_{2}=-y_{1}
\end{aligned}
$$

Because of this symmetry, eqns (5) and (6), (10) and (11), (12) and (13) are identical to each other. There are only two unknowns which we choose to be

$$
\begin{aligned}
& X=\frac{1}{1+x_{1}}=\frac{1}{1+e \cos f_{1}} \\
& Y=\sqrt{\frac{p_{1}}{p}}=\sqrt{\frac{p_{1}}{r_{1}\left(1+x_{1}\right)}}
\end{aligned}
$$

Using relations (17) and the definition of $X$, we write eqn (4) as

$$
\tan \phi_{1} \tan (\Delta / 2)=1 /(1+X)
$$

Similarly, we write eqn (5) as

$\left[y_{1}-(1 / X) \tan \phi_{1}\right]\left[1-\tan \phi_{1} \tan (\Delta / 2)\right]=\tan \phi_{1}$

Next, we transform eqns (10) and (12) into

$$
\tan \phi_{1}=[X /(1-Y)]\left[y_{1}-(1 / Y) e_{1} \sin \theta_{1}\right]
$$

and

$$
y_{1}=[(X-1) / X] \tan (\Delta / 2)
$$

To these equations we add eqn (7) written as

$$
e_{1} \cos \theta_{1}=Y^{2} / X-1 \text {. }
$$

To solve the system, we first eliminate $y_{1}$ between eqns (20) and (22) to obtain

$$
\begin{aligned}
& {\left[(X-1) \tan (\Delta / 2)-\tan \phi_{1}\right]} \\
& \quad \times\left[1-\tan \phi_{1} \tan (\Delta / 2)\right]=X \tan \phi_{1}
\end{aligned}
$$

By using eqn (19) for simplification, this results in

$$
(X-1) \tan (\Delta / 2)=(2+X) \tan \phi_{1}
$$

Combining eqns (19) and (24), we have the equation for the thrust angle

$$
\tan ^{2} \phi_{1}=\frac{(X-1)}{(X+1)(X+2)}
$$

which shows that the maximum possible thrust angle is such that $\tan \phi_{\max }=\sqrt{3}-\sqrt{2}, \phi_{\max }=17.632194^{\circ}$. If we use eqn (22) in eqn (21), with the aid of eqns (24) and (25), we obtain

$$
e_{1} \sin \theta_{1}=\frac{Y(X+Y+1)}{X} \sqrt{\frac{(X-1)}{(X+1)(X+2)}}
$$

Squaring eqns (23) and (26) and adding, we have

$$
e_{1}^{2}=\frac{Y^{2}(X+Y+1)^{2}(X-1)}{X^{2}(X+1)(X+2)}+\frac{\left(Y^{2}-X\right)^{2}}{X^{2}}
$$

On the other hand, using the same two equations to eliminate $e_{1}$ and noticing that $\theta_{1}=f_{1}+(\alpha / 2)$, we come up with another equation

$$
\begin{aligned}
&(X-1)\left[X Y^{2}-(X+1) Y-X(X+1)\right] \tan (\alpha / 2) \\
&=(X+1)\left[(2 X+1) Y^{2}\right. \\
&\left.+\left(X^{2}-1\right) Y-X(X+2)\right] \\
& \times \sqrt{\frac{(X-1)}{(X+1)(X+2)}}
\end{aligned}
$$

For given $e_{1}$ and $\alpha$, eqns (27) and (28) can be solved for $X$ and $Y$. The pertinent elements of the optimal transfer orbit can be deduced. In particular, the total minimum characteristic velocity for the two-impulse transfer can be obtained from eqn (16) written as

$$
\frac{\Delta V_{\mathrm{II}}}{\sqrt{\mu / p_{1}}}=\frac{\Sigma \Delta V_{i}}{\sqrt{\mu / p_{1}}}=\frac{2 Y(1-Y)}{X \cos \phi_{1}}
$$

This minimum cost for two-impulse transfer has to be compared with the cost via parabolic orbits. In this mode, called the $\mathbf{P}$ mode, the vehicle is sent into a parabolic orbit where the rotation can be achieved at large distance with infinitesimal cost. The last phase of the transfer is the return from parabolic orbit to the final elliptic orbit. The total cost for this transfer is

$$
\frac{\Delta V_{P}}{\sqrt{\mu / p_{1}}}=2\left[\sqrt{2\left(1+e_{1}\right)}-\left(1+e_{1}\right)\right]
$$

Now, if we assume that the planet around which the transfer is being performed is surrounded by an atmosphere with radius $R \leq r_{p_{1}}$, where $r_{p_{1}}$ is the perigee distance of the initial orbit, the following aeroassisted scheme may be considered. First, a decelerative impulse is applied tangentially at the apogee of the initial orbit to lower the perigee to the distance $R$. At this distance, atmospheric drag will act to progressively reduce the apogee without fuel consumption. Once the orbit is circularized, we can freely select the new direction for the apsidal line to coincide with the one of the final orbit. The final maneu- 
ver is a Hohmann transfer from circular orbit with radius $R$ to the final orbit. This aeroassisted transfer is independent of the rotation angle $x$ and hence may result in fuel savings for large $x$. The toal cost for this aeroassisted transfer can be easily computed to be

$$
\begin{array}{r}
\frac{\Delta V_{A E}}{\sqrt{\mu / p_{1}}}=2\left(1-e_{1}\right)-\sqrt{n\left(1+e_{1}\right)}+\left[n\left(1+e_{1}\right)\right. \\
\left.-2\left(1-e_{1}\right)\right] \sqrt{\frac{2}{n\left(1+e_{1}\right)+\left(1-e_{1}\right)}}
\end{array}
$$

where $n$ is the ratio of the perigee distance to the radius of the atmosphere

$$
n=r_{n_{1}} / R
$$

This aeroassisted mode is called the AE mode for the reason that the reentry trajectory is elliptic. It should be noticed that a complete circularization for rotation is not necessary. This is because of the fact that although the rotation is effected without cost when the orbit is circular, additional fuel is required in the last phase for climbing to the final elliptic orbit. The condition for this trade-off will be given in explicit form in the next section.

Another aeroassisted mode, called the AP mode, can be considered as follows. The vehicle is initially propelled into a parabola where the rotation can be effected without fuel consumption, just as for the $\mathrm{P}$ mode. Then, after the rotation, an infinitesimal impulse is applied to return the vehicle for grazing the atmosphere. In subsequent orbit decay, the apogee is reduced to the level of the final orbit where an accelerative impulse is applied to bring the perigee from $\mathrm{R}$ to the final value $r_{p_{1}}$. The total cost for this aeroassisted transfer is computed to be

$$
\begin{aligned}
\frac{\Delta V_{\mathrm{AP}}}{\sqrt{\mu / p_{1}}}=\sqrt{2\left(1+e_{1}\right)} & -2 e_{1}-\left(1-e_{1}\right) \\
& \times \sqrt{\frac{2}{n\left(1+e_{1}\right)+\left(1-e_{1}\right)}}
\end{aligned}
$$

We have compared the different modes of transfer and the results of this investigation are summarized in Figs 4-6. First, the optimal pure propulsive transfer is assessed, and it depends on the eccentricity $e_{1}=e_{2}$ of the terminal orbits and the rotation angle $\alpha$. In Fig. 4, the dashed line is the line of equality

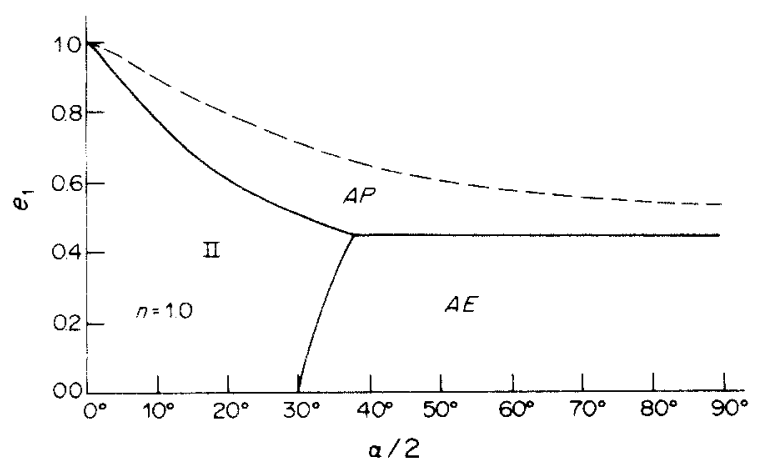

Fig. 4. Optimal regions for $n=1$

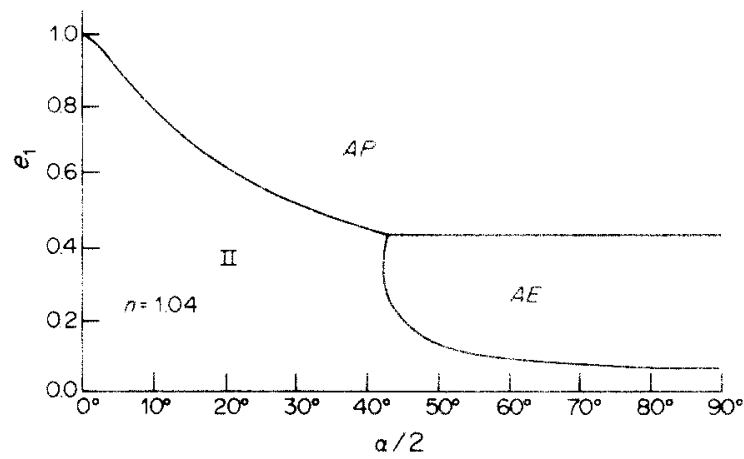

Fig. 5. Optimal regions for $n=1.04$

between the two-impulse transfer, called the II mode and the parabolic mode. Above this line, the $P$ mode is better, and in the $\left(\alpha / 2, e_{1}\right)$ space it is seen that the II mode is optimal over a large region.

The figure also displays the optimal regions for the aeroassisted transfer in the most favorable case of $n=1$, that is when the perigees of the terminal orbits are slightly above the sensible atmosphere. Then in the AE mode, the circularization and the rotation are achieved without fuel consumption, and the cost for this transfer is simply the cost for the Hohmann transfer from the circular orbit with radius $R$ to the final orbit. As for the AP mode, its cost is exactly the half of the cost for the P mode. Hence the P mode is eliminated when aeroassisted transfer is introduced, and we have the result as shown on the graph. The optimal region for the II mode is severely restricted. The AE mode and the AP mode are equal when

$$
e_{1}=\frac{1}{4}(2 \sqrt{2}-1)=0.45710678
$$

In the figure, it should be noticed that the $\alpha / 2$ axis (that is the line $e_{1}=0$ ) is the line of equality between the II mode and the AE mode, since at this limit the cost is zero for both these modes. This brings up an interesting feature for the aeroassisted transfer between orbits with low perigees as shown in Fig. 5 for the case where $n=1.04$. As the perigee increases, the region of optimality for the II mode expands at the expense of the AP mode and the AE mode. As a general rule, aeroassisted transfer is optimal when the eccentricity is high and/or the transfer angle is large. But in the case of low perigee, $n \approx 1$, there exists a range of rotation angles $\alpha$, where as we increase the eccentricity from zero, the optimal mode changes from the II mode to the AE mode and comes back to the II mode before changing to the AP mode at high values of the eccentricity.

When we increase the ratio $n=r_{p_{1}} / R$ from unity to higher values the optimal regions change as shown in Fig. 6. The two-impulse mode always exists.

In Fig. 6a, we have the case where $1<n<1.181962$. Aeroassisted transfer consists of the AP mode and the AE mode with high eccentricity for the AP mode. Let

$$
x=\sqrt{\frac{2 n\left(1+e_{1}\right)}{n\left(1+e_{1}\right)+\left(1-e_{1}\right)}}
$$



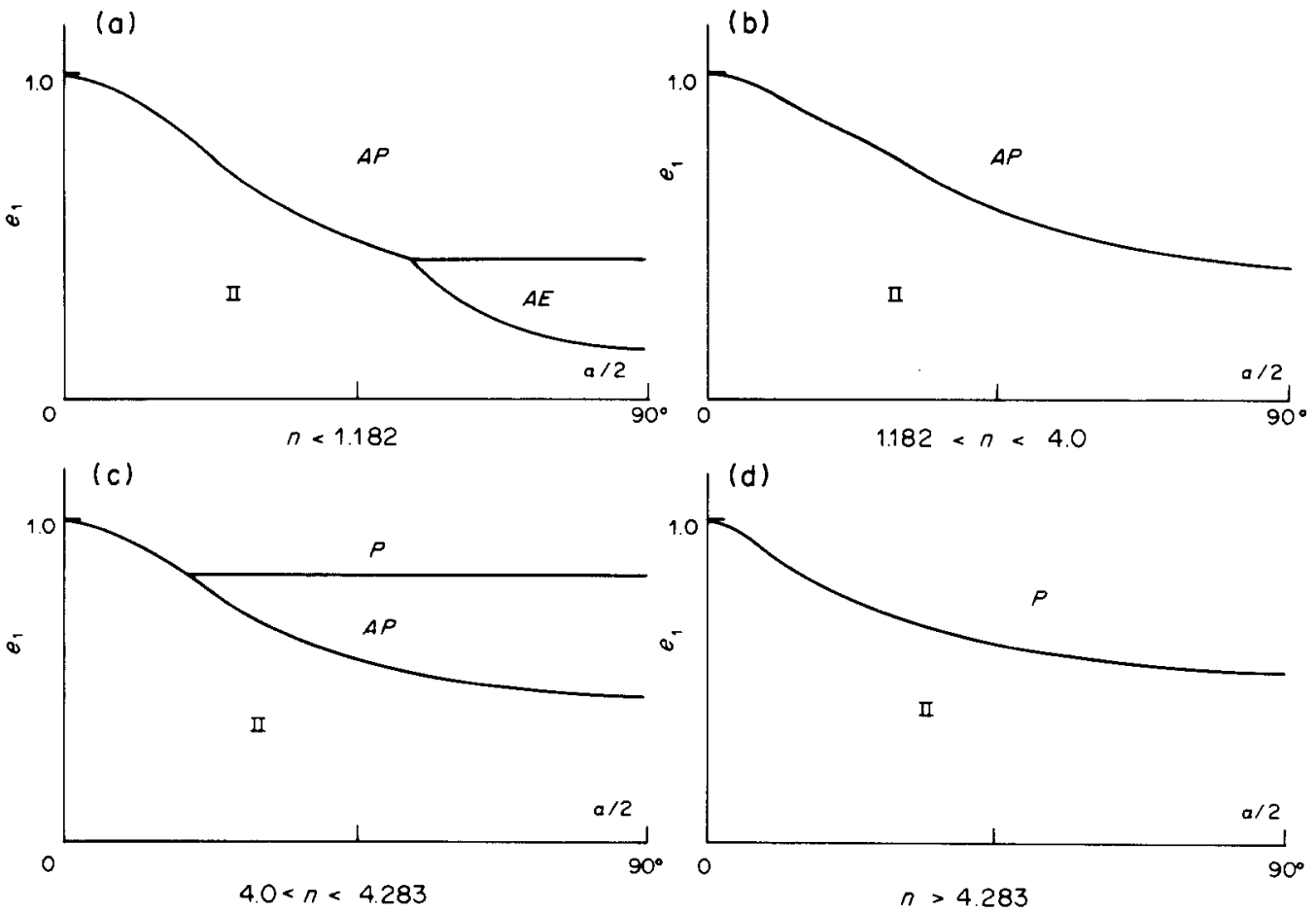

Fig. 6. Behavior of the optimal regions in terms of $n$.

For each value of $n$, by writing the equality of the costs between the AP mode and the AE mode, we have the cubic equation

$$
2 \sqrt{\frac{2}{n}}=\frac{4 x^{3}-4 x^{2}-5 x+4}{2 x^{2}-x-2}
$$

Upon solving for $x$, from eqn (35) we deduce the value of $e_{1}$ where the two modes are equal

$$
e_{1}=\frac{(n+1) x^{2}-2 n}{2 n-(n-1) x^{2}}
$$

When $n=1$, we have the exact solution (34).

In Fig. 6a, below a certain eccentricity, aeroassisted transfer is no longer optimal. This value is obtained by comparing the AE mode with the II mode when $\alpha=180^{\circ}$. This corresponds to the maximum value for the cost of the two-impulse transfer

$$
\frac{\Delta V_{\mathrm{II}}}{\sqrt{\mu / p_{1}}}=2\left[\sqrt{1-e_{1}}-\left(1-e_{1}\right)\right]
$$

Equating the two equations (31) and (38), we have the equation for computing $x$.

$$
\begin{aligned}
& 2 \sqrt{2-x^{2}}+\left(4+x-3 x^{2}\right) \\
& =4\left(2-x^{2}\right) \sqrt{\frac{2 n}{2 n-(n-1) x^{2}}}
\end{aligned}
$$

The value of the limiting eccentricity is given by eqn (37). As $n$ increases, the AE region decreases and disappears when

$$
\begin{aligned}
& x=1.19785478 \\
& n=1.18196153 \\
& e_{1}=0.36468763
\end{aligned}
$$

This solution is obtained by solving the two equations (36) and (39) for $x$ and $n$.

For the value of $n$ in the range $1.182<n<4$ besides the II mode, only the AP mode is available (Fig. 6b). At $\alpha=180^{\circ}$, the II mode and the AP mode are equal when

$$
\sqrt{2}+\frac{(c-3)}{\sqrt{c+1}}=\sqrt{c}-\frac{1}{\sqrt{n c+1}}
$$

where

$$
c=\left(1+e_{1}\right) /\left(1-e_{1}\right)
$$

When $n>4$, the $P$ mode begins to appear at high eccentricity at the expense of the AP mode (Fig. 6c). We have the equality of these two modes when

$$
e_{1}=\left(4+4 n-n^{2}\right) /\left(4-4 n+n^{2}\right)
$$

In terms of $c$, we have

$$
c=4 / n(n-4)
$$

The AP mode disappears at a value of $n$ satisfying both the two eqns (41) and (44). By eliminating $c$, we have a cubic equation

$$
n^{3}+4 n^{2}-28 n-32=0
$$

with the solution

$$
\begin{aligned}
& n=4.28267223 \\
& e_{1}=0.53533403
\end{aligned}
$$

When $n>4.283$, aeroassisted transfer is no longer optimal (Fig. 6d). 


\section{OPTIMAL TRANSFER FROM NEARLY CIRCULAR ORBIT}

It has been mentioned that in the orbit contraction process the oribit needs not be circularized. The reason is that although the rotation is made free of fuel consumption when the orbit is circular, circularizing involves additional cost to increase the energy from low circular orbit to the final energy. This trade off requires finding the optimal configuration of the contracting orbit. This will be done numerically in the next section with a specific example. Here we shall give an analytic solution to the equations in Section 2 for the case where the eccentricity of the initial orbit is small, that is $e_{1}=t$. From the solution to the first order in $t$, we casily derive the condition for noncircularization.

The perigee of the first orbit is fixed at the top of the atmosphere at distance $r_{n}=R=1 / d$. Hence wc have

$$
p_{1}=(1+6) d d
$$

We assume that $t \ll 1$. The other given quantities, $e_{2}$, $p_{2}$ and $x$, are arbitrary. It is convenient to change the notation with the definition

$$
s_{i}=1 / r_{i}, \quad q_{1}=1 / p, \quad q=1 / p
$$

When the initial orbit is circular, $t=0$, and we have the Hohmann transfer used as a reference solution

$$
\begin{array}{ll}
\theta_{1}=x, \quad \theta_{2}=\pi, \quad \Delta=\pi \\
f_{1}=0, \quad f_{2}=\pi, \quad \phi_{1}=0, \quad \phi_{2}=0
\end{array}
$$

To ease the notation. in the equations in the second section, we use the symbols $\bar{s}_{i}, \bar{\omega}, \bar{q}, \bar{\theta}_{i}, \bar{e}, \bar{f}_{i}, \bar{\phi}_{i}$ to denote the unknowns and calculate to the first order in $\epsilon$

$$
\begin{gathered}
\bar{s}_{i}=s_{i}+\epsilon s_{i}^{\prime} \\
\cdots \\
\bar{\phi}_{i}=\phi_{i}+\epsilon \phi_{i}^{\prime}
\end{gathered}
$$

where $s_{i}, \ldots, \phi_{i}$ denote the reference solution. With this, we write eqns (1) as

$$
\begin{aligned}
& s_{1}+\epsilon s_{1}^{\prime}=\frac{d}{(1+\epsilon)}\left[1+\epsilon \cos \left(\alpha+\epsilon \theta_{1}^{\prime}\right)\right] \\
& s_{2}+\epsilon s_{2}^{\prime}=q_{2}\left[1-e_{2} \cos \epsilon \theta_{2}^{\prime}\right]
\end{aligned}
$$

Identifying the coefficients of $\epsilon^{0}, \epsilon$, we obtain

$$
\begin{aligned}
& s_{1}=d, \quad s_{1}=-2 d \sin ^{2}(x / 2) \\
& s_{2}=k d, \quad s_{2}^{\prime}=0
\end{aligned}
$$

where

$$
k=\left[q_{2}\left(1-e_{2}\right)\right] / d=r_{p_{1}} / r_{a_{2}}
$$

is the ratio of the perigee distance of the first orbit to the apogee distance of the second orbit.

Next, we write eqn (7) as

$$
\begin{aligned}
& s_{1}+\epsilon s_{1}^{\prime}=\left(q+\epsilon q^{\prime}\right)\left[1+\left(e+\epsilon e^{\prime}\right) \cos \epsilon f_{1}^{\prime}\right] \\
& s_{2}+\epsilon s_{2}^{\prime}=\left(q+\epsilon q^{\prime}\right)\left[1-\left(e+\epsilon e^{\prime}\right) \cos \epsilon f_{2}^{\prime}\right]
\end{aligned}
$$

We easily deduce the solution

$$
\begin{aligned}
& q=(d / 2)(1+k), \quad q^{\prime}=-d \sin ^{2}(\alpha 2) \\
& e=(1-k)(1+k), \quad e-\frac{4 k}{11+k)^{2}} \sin ^{2}(\alpha / 2)(55)
\end{aligned}
$$

As seen from eqn (16), this is sufficient for evaluating the characteristic velocity to the first order in $t$. Define the new dimensionless characteristic velocities as

$$
\frac{\Delta V_{i}}{\sqrt{\mu / r_{r}}}=\frac{\Delta r_{i}}{\sqrt{\mu d}}=\Delta t
$$

From eqn (16), we have

$$
\begin{aligned}
& \Delta c_{1}=\left(\sqrt{\frac{2}{1+k}}-1\right)+c\left[\left(2-\frac{(1+2 k)}{(1+k)} \sqrt{\frac{2}{1+k}}\right)\right. \\
& \left.\times \sin ^{2} \frac{\alpha}{2}-\frac{1}{2}\right] \\
& \Delta v_{2}=k\left(\sqrt{\frac{\left(1-e_{2}\right)}{k}}-\sqrt{\frac{2}{1+k}}\right)-k \sqrt{\frac{2}{(1+k)}} \\
& \times \sin ^{2}(x / 2)
\end{aligned}
$$

The total characteristic velocity for a transfer from nearly circular orbit is

$$
\Delta v=\Delta v_{\mathrm{H}}+\dot{\partial}(\Delta v)
$$

where $\Delta v_{\mathrm{H}}$ is the cost for the Hohmann transfer

$$
\Delta v_{\mathrm{H}}=(1-k) \sqrt{\frac{2}{(1+k)}}+\sqrt{k\left(1-e_{2}\right)} \cdots
$$

and the perturbed fuel consumption is represented by

$$
\delta(\Delta v)=\epsilon\left[-\frac{1}{2}+\sqrt{2} \sin ^{2} \frac{x}{2}\left(\sqrt{2}-\frac{(1+3 k)}{\sqrt{(1+k)^{3}}}\right)\right]
$$

If $\delta(\Delta v)<0$, it is advantageous for the first orbit to remain elliptic. Hence the condition for a complete circularization to be nonoptimal is that

$$
\sin ^{2} \frac{x}{2}\left[\sqrt{2}-\frac{(1+3 k)}{\sqrt{(1+k)^{3}}}\right]<\frac{\sqrt{2}}{4}
$$

In particular, if $k>0.043584$, the condition is satisfied for any value of $\alpha$.

To obtain a complete solution, we shall evaluate the angular variables $\theta_{i}^{\prime}, f_{i}^{\prime}, \phi_{i}^{\prime}$ and $\omega^{\prime}$.

We notice that

$$
\bar{\Delta}=\pi+\epsilon \Delta^{\prime}=\pi+\epsilon\left(\theta_{2}^{r}-\theta_{1}\right)=\pi+\epsilon\left(f_{2}-f_{i}\right)
$$

Then, to the first order in $\varepsilon$, from definition (9)

$$
K_{1}=-\left(\phi_{1}^{\prime}+\phi_{2}^{\prime}\right) / \Delta^{\prime}=K, \quad K_{2}=-K
$$

To the first order in $\epsilon$, we linearize eqn (4) to be

$$
(2+e) K-(1+e)=-(2-e) K-(1-e)
$$

Solving for $K$ with solution (55) for $e$, we obtain

$\theta_{2}^{\prime}-\theta_{1}^{\prime}=\Delta^{\prime}=-\frac{2(1+k)}{(1-k)}\left(\phi_{1}^{\prime}+\phi_{2}^{\prime}\right)=f_{2}^{\prime}-f_{i}^{\prime}$ 
Equating the coefficients of $\epsilon$ in eqns (5), (6), (10) and (11), and noticing that $2 K=e$, we have

$$
\begin{aligned}
{\left[e f_{1}^{\prime}-(1+e) \phi_{1}^{\prime}\right](2-e) } & =2 \phi_{1}^{\prime} \\
{\left[e f_{2}^{\prime}+(1-e) \phi_{2}^{\prime}\right](2+e) } & =-2 \phi_{2}^{\prime} \\
\frac{s_{1}}{q}\left(1-\sqrt{\frac{q}{d}}\right) \phi_{1}^{\prime} & =e f_{1}^{\prime}-\sqrt{\frac{d}{q}} \sin \alpha \\
\frac{s_{2}}{q}\left(1-\sqrt{\frac{q}{q_{2}}}\right) \phi_{2}^{\prime} & =-e f_{2}^{\prime}+e_{2} \theta_{2}^{\prime} \sqrt{\frac{q_{2}}{q}}
\end{aligned}
$$

Equations (65) and (67) can be solved for $f_{1}^{\prime}$ and $\phi_{1}^{\prime}$. We have

$$
\phi_{1}^{\prime}=\frac{(1+3 k) \sin \alpha}{(1+3 k)+\sqrt{2(1+k)^{3}}}
$$

and

$$
f_{1}^{\prime}=\frac{2\left(k^{2}+5 k+2\right) \sin \alpha}{(1-k)\left[(1+3 k)+\sqrt{2(1+k)^{3}}\right]}
$$

Then eqns (64) and (66) give $f_{2}^{\prime}$ and $\phi_{2}^{\prime}$. We have

$$
\phi_{2}^{\prime}=\frac{(3+k)(1+2 k) \sin \alpha}{(2+k)\left[(1+3 k)+\sqrt{2(1+k)^{3}}\right]}
$$

and

$$
f_{2}^{\prime}=-\frac{2\left(2 k^{2}+5 k+1\right)(1+2 k) \sin \alpha}{(1-k)(2+k)\left[(1+3 k)+\sqrt{2(1+k)^{3}}\right]}
$$

From eqn (68) we obtain

$$
\begin{gathered}
e_{2} \theta_{2}^{\prime}=-\left[\left(1-e_{2}\right)+\frac{(1+k)}{(3+k)}\right. \\
\left.\sqrt{\frac{2(1+k)\left(1-e_{2}\right)}{k}}\right] \phi_{2}^{\prime} .
\end{gathered}
$$

The variation in the transfer angle is

$$
\Delta^{\prime}=-\frac{2(1+k)\left(5 k^{2}+14 k+5\right) \sin \alpha}{(1-k)(2+k)\left[(1+3 k)+\sqrt{2(1+k)^{3}}\right]}
$$

From this we deduce $\theta_{1}^{\prime}=\theta_{2}^{\prime}-\Delta^{\prime}$ and also the variation of the argument of the perigee of the transfer orbit from eqn (15) as $\omega^{\prime}=\theta_{1}^{\prime}-$ $f_{1}^{\prime}=\theta_{2}^{\prime}-f_{2}^{\prime}$.

The linearized solution we have derived is for the case where $r_{p_{2}}>r_{p_{1}}=R$. In the reference solution, $\phi_{2}=0$ and the second impulse is an accelerative impulse. In the case where $r_{p_{1}} \geqslant r_{p_{2}}$, in the reference solution $\phi_{2}=\pi$, and the second impulse is a decelerative impulse. Again, we use $r_{p_{1}}$ as the reference distance to obtain the linearized solution applicable to this case. For $r_{i}, p$ and $e$, we have the same solution, eqns (52) and (55) as before. For the characteristic velocities as given by eqn (57), $\Delta v_{1}$ is the same but for $\Delta v_{2}$, we must change the sign on the right hand side. The characteristic velocity for the Hohmann transfer is now

$$
\Delta v_{\mathrm{H}}=\sqrt{2(1+k)}-\sqrt{k\left(1-e_{2}\right)}-1
$$

For a slightly elliptic initial orbit with eccentricity $e_{1}=\epsilon$, the perturbed fuel consumption is

$$
\delta(\Delta v)=\epsilon\left[-\frac{1}{2}+\left(2-\sqrt{\frac{2}{1+k}}\right) \sin ^{2} \frac{\alpha}{2}\right]
$$

Hence we now have the condition for noncircularization

$$
\left(2-\sqrt{\frac{2}{1+k}}\right) \sin ^{2} \frac{\alpha}{2}<\frac{1}{2}
$$

The formulas for the angular variables $\theta_{i}^{\prime}, f_{i}^{\prime}, \phi_{i}^{\prime}$ and $\omega^{\prime}$ are different. First we have

$$
\phi_{i}^{\prime}=\frac{\sin \alpha}{1+\sqrt{2(1+k)}}
$$

From this, we compute the other variables

$$
\begin{aligned}
& f_{1}^{\prime}=\frac{2(2+k)}{(1-k)} \phi_{1}^{\prime} \\
& \phi_{2}^{\prime}=-\frac{(1+2 k)}{(2+k)} \phi_{1}^{\prime} \\
& f_{2}^{\prime}=\frac{2(1+2 k)^{2}}{(1-k)(2+k)} \phi_{1}^{\prime} .
\end{aligned}
$$

The perturbation in the transfer angle is

$$
\Delta^{\prime}=-\frac{6(1+k)}{(2+k)} \phi_{i}^{\prime}
$$

Then, as before, we have $\theta_{1}^{\prime}=\theta_{2}^{\prime}-\Delta^{\prime}, \omega^{\prime}=\theta_{1}^{\prime}-f_{1}^{\prime}$, but now with

$$
\begin{aligned}
e_{2} \theta_{2}^{\prime}= & \frac{(1+2 k)}{(2+k)} \\
& \times\left[\left(1-e_{2}\right)+\sqrt{\frac{2(1+\mathbf{k})\left(1-e_{2}\right)}{k}}\right] \phi_{1}^{\prime}
\end{aligned}
$$

As will be tested in the next section, besides the exact condition for noncircularization as given explicitly in eqns (61) or (77), the linearized solutions are fairly accurate for small values of $\epsilon$.

\section{NUMERICAL EXAMPLES}

As numerical examples, we first consider the problem of rotation of an orbit through an angle $\alpha=80^{\circ}$. For the terminal orbits, we have $p_{1}=p_{2}=1.5$ and $e_{1}=e_{2}=0.5$. Hence $r_{\mathrm{p}_{1}}=p_{1} /\left(1+e_{1}\right)=1=r_{\mathrm{p}_{2}}$. For a pure propulsive transfer, we obtain $\theta_{1}=145.699^{\circ}$, $\theta_{2}=360^{\circ}-\theta_{1}=214.301^{\circ}$ and $p=2.28925$. This requires a characteristic velocity $\Delta v_{\mathrm{II}}=0.22759$. It should be noticed that in this section, we use the circular speed at the distance $r_{p_{1}}=1$ as the reference speed. The parabolic transfer costs $\Delta v_{p}=0.37894$. Hence, for pure propulsive transfer, the two-impulse mode is optimal. If we assume that the perigees of the terminal orbits are at the realm of the atmosphere, then circularization of the initial orbit is effected without fuel consumption and the cost for aeroassisted transfer is the cost for the Hohmann transfer as computed from eqn (75) with $k=1 / 3$. This gives $\Delta v_{\mathrm{AE}}=0.22474$. Hence this aeroassisted transfer by complete circularization is better although the fuel 


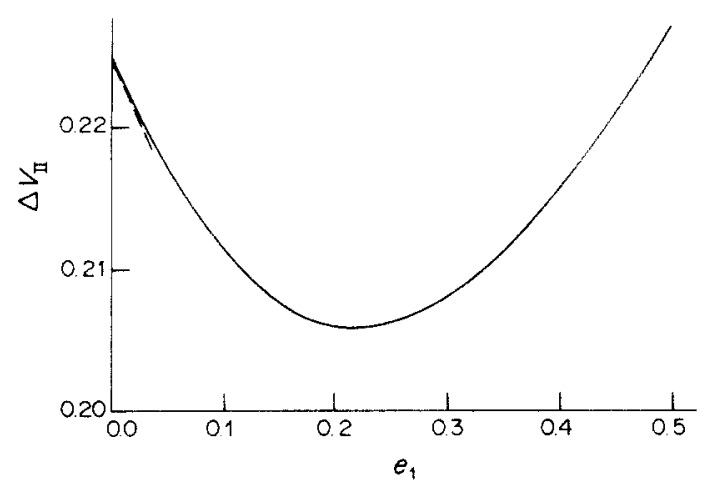

Fig. 7. Characteristic velocity of the two-impulse transfer as function of the initial eccentricity.

saving is small. As seen in Fig. 4, the optimal mode is the AP mode which costs $\Delta v_{\mathrm{AP}}=0.18947$, but is not practical for finite time transfer.

Now, if we check the condition (77), we find that complete circularization is non optimal up to the value $\alpha=106.852^{\circ}$. Therefore, $\Delta v_{\mathrm{AE}}$ can be reduced by stopping the orbit decay process at a certain optimal value $e_{1}^{*}>0$ of the starting elliptical orbit. The minimum cost for aeroassisted transfer will be the cost for the two-impulse transfer between this orbit and the final orbit. With $p_{1}=1+e_{i}, p_{2}=1.5$, $e_{2}=0.5$ and $\alpha=80^{\circ}$ and using the equations in the second section, we have solved the problem for several values of $e_{1}$ from $e_{1}=0.5$ to $e_{1}=0$. The total characteristic velocity is plotted versus $e_{1}$ in Fig. 7. It has been found that the optimal value of $e_{1}$ is $e_{1}^{*}=0.22$. The corresponding minimum cost for aeroassisted transfer is $\Delta v_{\mathrm{AE}}=0.20607$. As compared to the pure propulsive transfer, this amounts to a fuel savings of about $9.5 \%$.

In the figure, we have plotted in dashed line the characteristic velocity using the linearized theory for small eccentricity as given by eqns (75) and (76). The line is in fact the tangent to the curve displaying the exact solution, and as such, it correctly predicts the nonoptimality of the solution by circularization.

As another example, we consider the case of transfer from an initial orbit with $p_{1}=1.442, e_{1}=0.4$ to a final orbit with $p_{2}=1.560, e_{2}=0.5$ with a rotation of apsidal line of $\alpha=120^{\circ}$. With this, we have $r_{p_{1}}=1.03$, $r_{a_{1}}=2.40333, a_{1}=1.71667, \quad r_{p_{2}}=1.04, \quad r_{a_{2}}=3.12$, $a_{2}=2.08$. Hence this is the case of a transfer from a low-energy orbit to a high-energy orbit. Here the radius of the atmosphere is the unit distance and the circular speed at this distance is the unit speed.

For the pure propulsive transfer by two impulses we obtain the solution $\theta_{1}=154.9832^{\circ}, \theta_{2}=$ $197.4696^{\circ}, p=2.48454$, with a total characteristic velocity $\Delta v_{\mathrm{II}}=0.27650$. This is the optimal solution, since the transfer via parabolic orbits requires much higher cost. For aeroassisted transfer using the $\mathrm{AE}$ mode, we first use a decelerative impulse at the apogee of the initial orbit to reduce the perigee to the distance $r_{\mathrm{p}}=R=1$. This necessitates an impulse with magnitude $\Delta v_{1}=0.00517$. With complete circular- ization the remaining cost for the transfer is the cost for Hohmann transfer from low circular orbit to the final orbit such that $\Delta v_{2}+\Delta v_{3}=0.23068+0.00587$ The total cost for aeroassisted transfer is then $\Delta v_{\mathrm{AE}}=\Delta v_{1}+\Delta v_{2}+\Delta v_{3}=0.24172$. This represents a fuel saving of $12.6 \%$, which is significant considering the fact that this is a transfer from a low-energy orbit to a high-energy orbit. Since in this case, $k=1 / 3.2$, by condition (61), complete circularization is nonoptimal. It has been found that, during the decay process for reducing the eccentricity of the initial orbit, the best value to start the two-impulse transfer to the final orbit is $e_{1}^{*}=0.026$. From this value, the cost for the transfer to the final orbit including the first impulse to initiate the decay process is $\Delta v_{\mathrm{AE}}^{*}=0.23212$. This represents a saving of $16.1 \%$ as compared to the pure propulsive maneuver. This transfer is optimal, since the AP mode requires a cost of $\Delta v_{\mathrm{AP}}=0.23348$.

In the orbit decay process, the two orbits $O_{1}$ and $\mathrm{O}_{2}$ become tangential at the value $e_{1}=0.0258$, and in the vicinity of this value there is a small range of $e_{\text {; }}$ where the two-impulse mode becomes the oneimpulse optimal mode. The numerical computation is quite sensitive to the initial guessed values of the unknowns $\theta_{1}, \theta_{2}$ and $p$. In this respect the linearized solutions we have derived are very helpful for the first estimate of the solution. Also, it should be noticed that in practice, instead of a multirevolution process to achieve the desired optimal value $e_{!}^{*}$. we can use a steeper atmospheric entry angle $\gamma_{t} \neq 0$ 10 realize this orbit in one atmospheric passage. The penalty in $\Delta V$ is of the order of $\gamma_{i}^{2}[7]$.

As a final note, for the general case of planar transfer between arbitrary elliptical orbits, when the eccentricities are high and the perigee of the final orbit is low, the AP mode is frequently optimal. The inconvenience is that the transfer time is large even if we approximate the parabolas by ellipses with high eccentricities. For that reason, the AE mode with one atmospheric passage is the most promising transfer scheme in aeroassisted technology.

\section{CONCLUSIONS}

This paper presents the solution for minimum-fuel. free-time transfer between coplanar elliptical orbits with the possible use of atmospheric passage to generate a braking force as a replacement of a decelerative impulse. The optimal pure propulsive, two-impulse transfer is first considered. It is shown that the solution is obtained by solving a set of three equations for three unknowns which are taken as the true anomalies $\theta_{1}$ and $\theta_{2}$ of the location of the impulses on the initial and the final orbits, respectively, and the semilatus rectum $p$ of the transfer orbit. Reduction of the general equations is made for the case of symmetrical transfer and a complete first order solution is provided for the case of transfer from nearly circular orbit. 
Next the aeroassisted transfer is discussed and a complete solution is presented for the case of the rotation of an orbit. Aeroassisted transfer is a fuel saving scheme because of the fact that atmospheric braking at the perigee can be used to circularize the orbit and in the circular configuration the orbit can be arbitrarily rotated without fuel consumption. Complete circularization is optimal only when the rotation angle is large. An explicit formula for evaluating this critical angle is provided.

Finally, a complete numerical example is presented to show that aeroassisted transfer can be optimal even in the case of a transfer from a low-energy orbit to a high-energy orbit.

Acknowledgement-This work was supported by the Jet Propulsion Laboratory under contract No. 956416 with Dr K. D. Mease as project manager.

\section{REFERENCES}

I. N. X. Vinh, Optimal control of orbital transfer vehicles. AIAA paper No. 83-2092 (1983).

2. G. D. Walberg, A survey of aeroassisted orbit transfer. AIAA J. Spacecraft 22, 3-18 (1985).

3. D. F. Lawden, Optimal Trajectories for Space Navigation. Butterworths, London (1963).

4. N. X. Vinh, Exact relations of optimum switching in the problem of impulsive transfer. J. Astronaut. Sci. 17, 337-364 (1970)

5. C. Marchal, Une expression des commutations sous forme explicite dans le problème des transferts optimaux entre orbites képlériennes. Rech. Aérosp. 1971, 1-7 (1971)

6. J. P. Marec, Optimal Space Trajectories. Elsevier, Amsterdam (1979).

7. N. X. Vinh, J. R. Johannesen and K. D. Mease, Explicit guidance of drag-modulated aeroassisted transfer between elliptical orbits. AIAA J. Guidance Control Dynam. 9, 274-280 (1986). 